SUBJECT INDEX

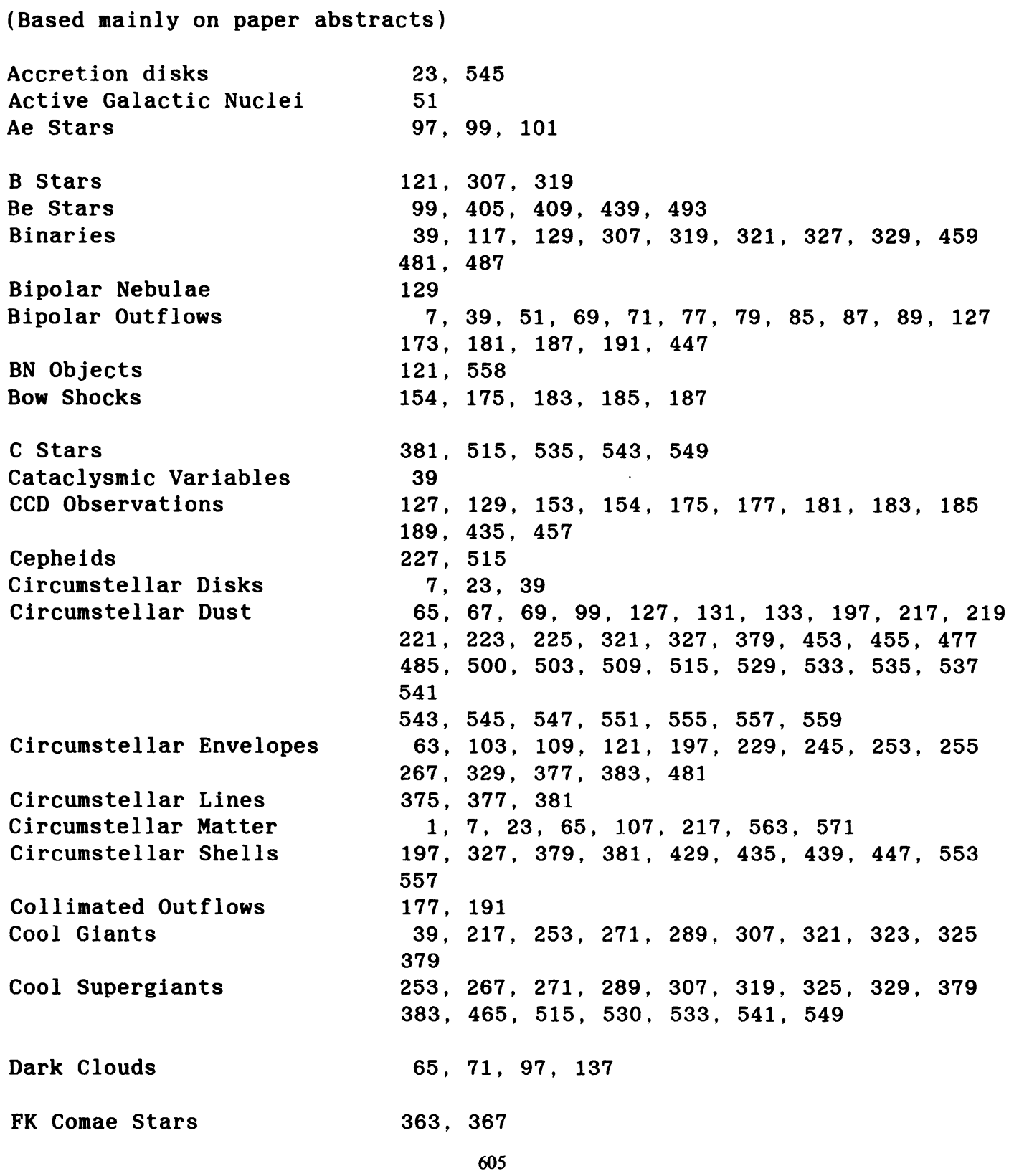


FU Orionis Stars

GGD Objects

Globular Clusters

Herbig-Haro Objects

HII Regions

$\mathrm{H}_{2} \mathrm{O}$ Masers

Hot Stars

Hybrid Stars

Infrared Interferometry

Infrared Maps

Infrared Photometry

Infrared Polarimetry

Infrared Radiation

Infrared Spectrophotometry

Infrared Surveys

Interferometry

Interstellar Gas

Interstellar Medium

IRAS Sources

IUE Observations

Jets

Low Mass Stars

Magallenic Clouds

Magnetic Fields

Masers

Mass Loss

Mira Stars

MM Radiation

Molecular Clouds

Molecular Lines

Molecular Spectra

Novae

OI Lines
67

125

357

$51,147,159,173,175,179,181,183,185$ $187,189,191,193$

121,451

141,267

$39,395,409,425,441,445,447,449,451$

453,509

$275,321,362$

$65,245,525$

183

$113,115,125$

115,127

69

113

$93,189,197$

81

$159,389,451$

$389,445,451$

$67,93,113,125,133,189,197,215,217$

$219,221,223,225,227,241,321,327,379$

382,413

499,512

$156,275,309,319,321,323,364,381,417$

$431,441,443,449,469,481,493$

$7,39,51,73,75,147,159,173,175,177$

$191,193,469,475,491,571$

$7,23,79,107,115,572$

$241,410,429,439,441,443$

$23,77,129,137,143,333,347$

$51,93,141,215,229,241,243,249,253$

$267, \quad 508$

$63,79,105,227,271,289,307,319,323$

325. $327,377,395,449,463$

$221,243,271,325,375,385,469,471,477$

535

$71,77,79,81,85,89,229,505,511$

$7,61,63,73,93,129$

$71,79,81,85,89,179$

227

39,547

101,189 
OH-IR Stars

Opacities

Orion Nebula

P Cygni Profiles

P Cygni Stars

Planetary Nebulae

Polarimetry

Pre-Main-Sequence Stars

Protoplanetary Nebulae

Protostars

Protostellar Clouds

R Stars
Radio Jets
Radio Lines
Radio Maps
Radio Stars
Radio Surveys
Reflection Nebulae
RS Cvn Stars
RV Tauri Stars
S Doradus Stars
Shocks
Sio Maser Stars
Speckle Interferometry
Star Formation
Stellar Chromospheres
Stellar Coronae
Stellar Flares
Stellar Transition Regions
Stellar Winds

Sub-MM Lines

Symbiotic Stars

\section{T Tauri Stars}

White Dwarfs

Wolf Rayet Stars

$X$-ray Sources

$\mathrm{X}$-ray Surveys

Young Stars
$215,229,241,243,245,247,249$

387

119

$103,431,433,439,449$

$425,433,435,465$

$39,431,499,501,503,505$

$129,131,133,135,137,471,475,485$

$39,63,79,99,101,103,105,107,115,125$

$39,507,509$

$7,39,51,65,121,141,189,572$

129,143

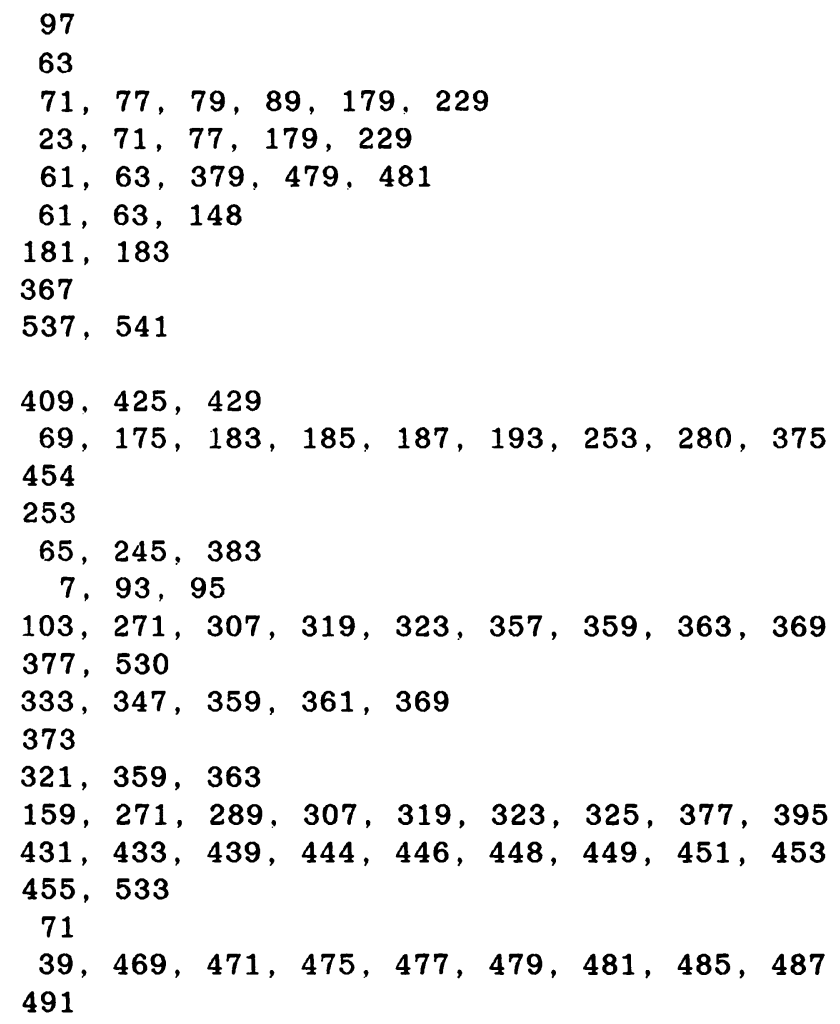

